\title{
Correlation between Areas of Ventricular Pre- excitation and Types of WPW QRS Patterns by Means of Computer Simulation of Ventricular Activation Sequence
}

\author{
Yoshihiro KoIKe, M.D.
}

\section{SUMMARY}

Computer simulation of the ventricular activation sequence of the WPW syndrome was attempted by producing fusion beats in which localized ventricular myocardium was activated earlier than the "normal excitation". It suggested that the site of pre-excitation was; i) at the posterior or the lateral free wall of the left ventricle adjacent to the $A-V$ groove in type $\mathrm{A}$, ii) at the antero-lateral base of the right ventricle in type $\mathrm{B}$, and iii) at the posterior paraseptal region of the right ventricle in type $\mathrm{C}$. A mild degree of pre-excitation at the left ventricular posterolateral region will result in type-B QRS complexes.

\section{Additional Indexing Words:}

WPW syndrome Ventricular pre-excitation Digital computer simulation Ventricular activation sequence Ventricular fusion beat

QINGE Durrer and Roosi) investigated the epicardial excitation of the $D$ ventricles in a patient with type-B Wolff-Parkinson-White (WPW) syndrome, the site of ventricular pre-excitation has been well identified by electrophysiological studies. ${ }^{2-81}$ In spite of being generally postulated that in type $\mathrm{B}$, the site of pre-excitation is located in the basal portion of the right ventricle, Lister and colleagues ${ }^{9}$ reported that, in a patient with type-B WPW syndrome, the earliest site of excitation was the posterior base of the left ventricle near the crux of the heart.

The attempt at surgical interruption of the accessory pathway was successful in subsequent abolition of both the electrocardiographic features of the syndrome and the episodes of tachycardia by Cobb et al, ${ }^{3)}$ but later studies did not necessarily succeed in surgical division of anomalous pathways, $\left.\left.{ }^{6}\right), 10\right), 11$ ) probably because localization of the earliest area of anomalous ventricular activation was not accurate even at surgery. It was reported that the site

From the First Department of Internal Medicine, School of Medicine, Nagoya University, 65 Tsurumai-cho, Showa-ku, Nagoya 466, Japan. The Research Institute of Environmental Medicine, Nagoya University, Nagoya, Japan.

Received for publication December 13, 1976. 
of ventricular pre-excitation could be approximately determined on the basis of body-surface electrocardiograms, and its precise localization could be highly facilitated at the non-invasive electrophysiologic studies. ${ }^{12}$ )

Electrocardiographic patterns analogous to the WPW syndrome were produced in experimental animals. ${ }^{13)-16)}$ However, these are not necessarily identical to the condition obtained in human subjects.

Therefore, in the present report, computer simulation of the ventricular activation sequence was attempted on the model of human ventricles to obtain WPW QRS complexes by producing fusion beats in which localized ventricular myocardium was activated earlier than the " normal excitation". Correlation was made between the area of ventricular pre-excitation and the configuration of the QRS complexes obtained, especially in lead $V_{1}$.

\section{Methods}

A digital computer was used to produce ventricular activation sequence in a model which was composed of 27,000 clusters of 3-mm cubic blocks and possessed simulated anatomical and electrophysiological properties of human ventricles. ${ }^{17}$ ) Based on this simulated heart model, QRS complexes in body-surface leads were mathematically constructed by the computer. ${ }^{18)}$

Pre-excitation was initiated at several sites of the ventricular model, with normal excitation started at the lower one third of the left interventricular septum after variable degrees of time delay. Thus, ventricular fusion beats were produced to simulate the ventricular activation sequence of the WPW syndrome. The following 5 locations were selected as the site of pre-excitation, i) the postero-basal portion of the left ventricle, ii) the anterolateral base of the right ventricle, iii) the right ventricular base near the posterior interventricular septum, iv) the pulmonary conus, and $v$ ) the upper part of the anterior interventricular sulcus. The time delay between the onset of pre-excitation and the beginning of normal septal activation varied from 10 to $90 \mathrm{msec}$. It will arbitrarily be designated as "pre-excitation time" in the following text.

\section{Results}

I. Pre-excitation at the postero-basal region of the left ventricle

1. The pre-excitation impulse was given to the subepicardial layer of the postero-lateral region of the left ventricle $50 \mathrm{msec}$ earlier than the onset of normal excitation. The ventricular activation sequence and constructed QRS complexes are shown in Fig. 1. At the early stages (stage 1 to 3 ) the activation wave front spread slowly through the ventricular myocardium in the epicardial to endocardial direction anteriorly to the right, ensuing in a small slurred upward deflection in the initial part of the QRS complex (delta wave) in leads $V_{1}, V_{3}$, and $V_{5}$. The pre-excitation impulse entered into the 


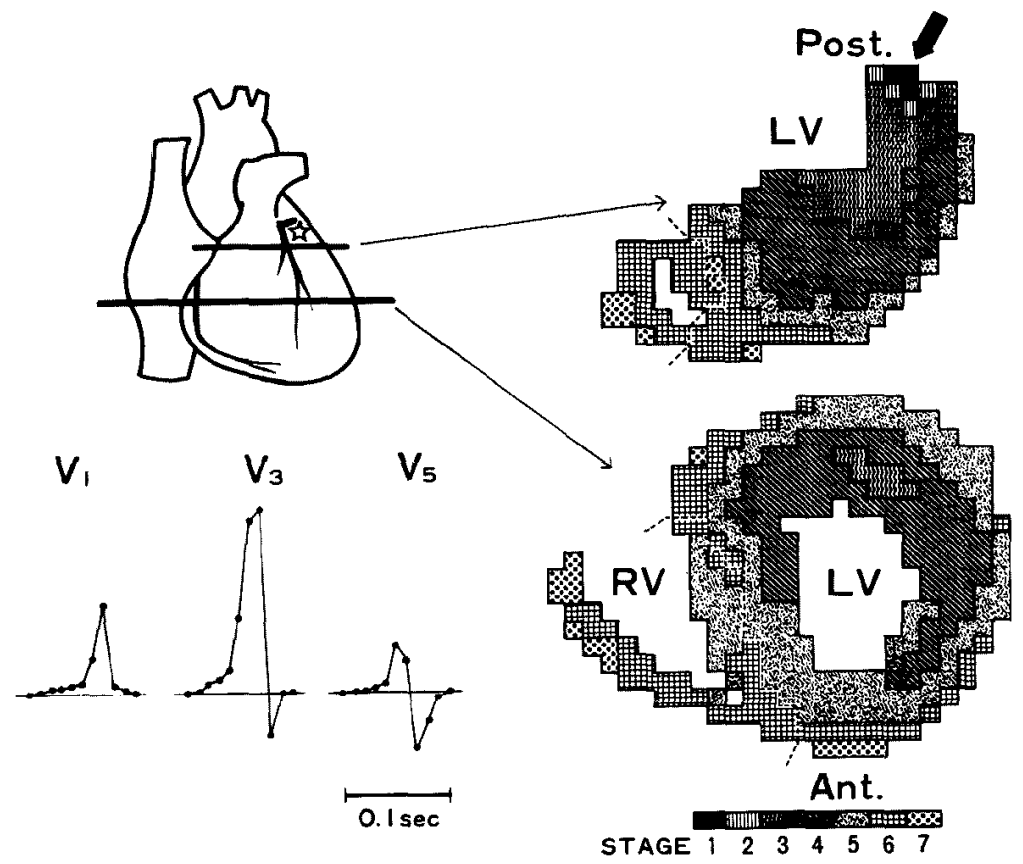

Fig. 1. Computer-simulated sequence of ventricular activation in preexcitation at the subepicardial layer of the posterobasal region of the left ventricle. The site indicated by arrow in the right upper panel was excited $50 \mathrm{msec}$ earlier than the onset of normal excitation. In the right panel the ventricular activation front at the $15-\mathrm{msec}$ interval are illustrated in the horizontal cross-section at the levels shown in the left upper panel. The QRS complexes in the chest leads $\left(V_{1}, V_{3}\right.$, and $\left.V_{5}\right)$ are compatible with those of the type-A WPW syndrome. The broken lines indicate the boundary where the activation wave fronts caused by the normal and pre-excitation impulses collide with each other.

Purkinje network $40 \mathrm{msec}$ after the onset of QRS (stage 3 ) and spread forward rapidly, resulting in a large $R$ wave in $V_{1}$ and $V_{3}$. At $50 \mathrm{msec}$ (stage 4 to 5 ) the left ventricle was activated circumferentially in the endo- to epicardial direction, while, in the right ventricle, the anterior papillary muscle and its adjacent myocardium began to be excited by the normally conducted impulse. In the right panel of Fig. 1 the activation wave fronts caused by the normal and pre-excitation impulses collided with each other at the boundary indicated by broken lines. Then, activation spread to the right ventricular free wall as well as the posterior margin of the interventricular septum. The apex was activated at the last stage. The constructed QRS complexes were compatible with those of the type-A WPW syndrome (Fig. 1).

Fig. 2 shows changes of QRS complexes in lead $V_{1}$ at various "preexcitation time". As pre-excitation time was shortened, the $\mathrm{R}$ wave in lead 


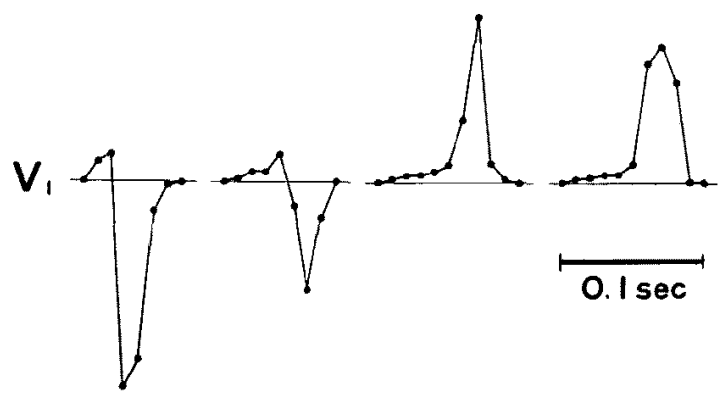

Fig. 2. QRS complexes in lead $V_{1}$ at various "pre-excitation time" with ventricular pre-excitation at the same location as in Fig. 1. The first QRS complex from the left was obtained when the ventricles were activated by the normal impulse. The second and third ones indicate fusion beats with the respective pre-excitation time of 30 and $50 \mathrm{msec}$. The second QRS complex should be classified as type $\mathbf{B}$ by definition, but the third is of type $A$ in configuration. The last is a QRS complex which was constructed when the ventricles were activated only by the pre-excitation impulse.

$\mathrm{V}_{1}$ became lower, the QRS duration shorter, and the delta wave smaller. In spite of pre-excitation at the postero-basal region of the left ventricle, the QRS complex obtained with pre-excitation time of $30 \mathrm{msec}$ was an $\mathrm{rS}$ configuration in lead $V_{1}$, which should be classified as type $B$ in Rosenbaum et al's classification. ${ }^{19)}$ It is to be emphasized that the delta vector remained nearly in the same direction irrespective of the degree of pre-excitation time.

2. When the pre-excitation impulse was given to the subendocardial layer of the left postero-basal region, it immediately invaded into the Purkinje network, and the activation wave front spread rapidly from right to left. The constructed QRS complex in lead $V_{1}$ showed various patterns such as $q R S$, $\mathrm{QR}$, or $\mathrm{qrsR}^{\prime}$ and was not accompanied by a significant initial slurring characteristic of typical WPW QRS complexes.

3. When pre-excitation was initiated at the subepicardial layer of the left basal region near the posterior interventricular sulcus, a QRS complex of $\mathrm{rSR}^{\prime}$ configuration with a definite delta wave was obtained, which was classified as an atypical type A. However, as pre-excitation time was shortened, type-B QRS complexes were observed.

II. Pre-excitation at the basal region of the right ventricle

1. The pre-excitation impulse was applied to the right lateral border neighboring the atrioventricular groove $40 \mathrm{msec}$ earlier than the onset of the normal excitation (Fig. 3). At the early stages the activation wave front spread slowly through the ventricular myocardium anteriorly to the left with a consequent small upright delta wave in lead $V_{1}$. At the middle stage (60 


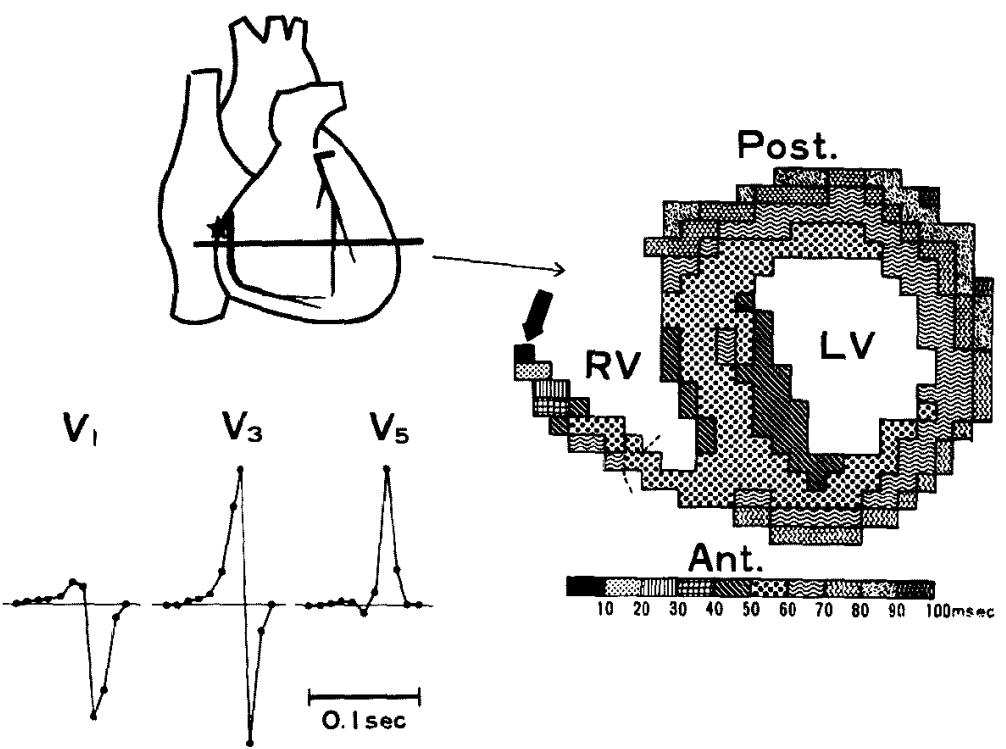

Fig. 3. Computer-simulated sequence of ventricular activation in preexcitation at the antero-lateral base of the right ventricle. The site indicated by arrow in the right panel was excited $40 \mathrm{msec}$ earlier than the onset of normal excitation. In the right panel the ventricular activation front at the 10-msec interval are illustrated in the horizontal cross-section of the heart. Precordial QRS complexes are compatible with those of the type-B WPW syndrome.

See legend of Fig. 1 for the explanation of the broken line.

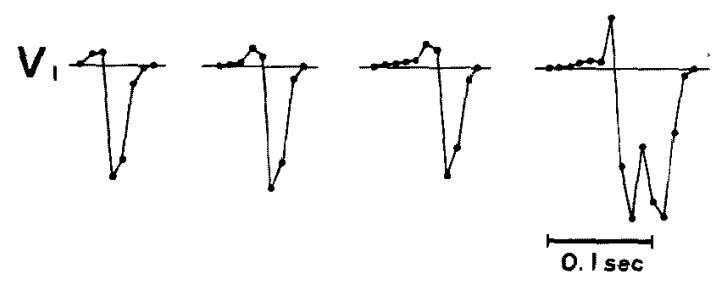

Fig. 4. QRS complexes in lead $V_{1}$ at various pre-excitation time with pre-excitation at the same location as in Fig. 3. The second and third QRS complexes from the lcft indicate fusion beats with the respective pre-excitation time of 20 and $40 \mathrm{msec}$. Irrespective of the degree of pre-excitation, they invariably show the type-B QRS complexes.

See legend of Fig. 2 for the left and right QRS complexes.

$\mathrm{msec}$ ) it proceeded rapidly to the left and, then, backward in the same fashion as the normal excitation. The pulmonary conus was activated at the middle stages $(60-70 \mathrm{msec})$, the apex relatively late $(80-90 \mathrm{msec})$, and the posterobasal region of the left ventricle at the last stage. The resultant QRS complex in lead $V_{1}$ was an $r S$ in configuration, which was characteristic of the type-B WPW syndrome. 


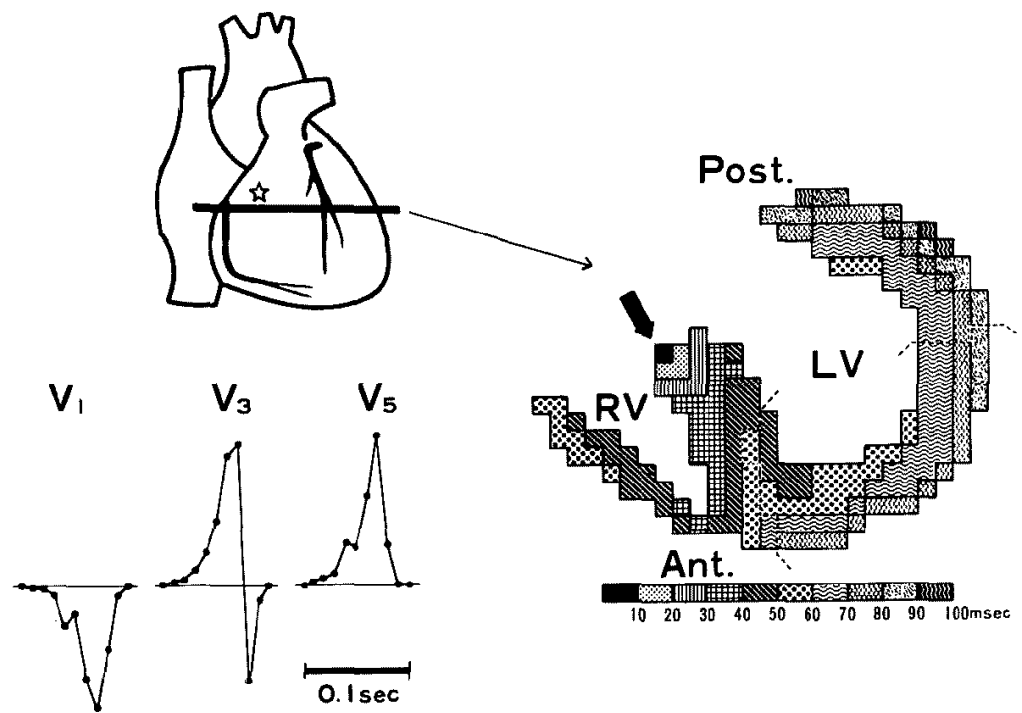

Fig. 5. Computer-simulated sequence of ventricular activation in preexcitation at the posterior paraseptal region of the right ventricle. The site indicated by arrow in the right panel was excited $40 \mathrm{msec}$ earlier than the onset of normal excitation. The simulated QRS complexes are those of type C. The lead $V_{1}$ shows a $Q S$ pattern with a notching on the descending limb.

See legend of Figs. 1 and 3 for the explanation of the broken lines and the ventricular activation wave front, respectively.

The QRS complexes in lead $V_{1}$ varied in configuration at various preexcitation time (Fig. 4). As pre-excitation time decreased, the delta wave became slightly smaller but remained almost unchanged in direction, and the QRS complex became abbreviated in duration but remained to be an $\mathrm{rS}$ pattern in lead $V_{1}$.

2. The pre-excitation impulse was given to the postero-basal region of the right ventricle near the interventricular septum $40 \mathrm{msec}$ earlier than the normal excitation (Fig. 5). At the early stage the excitation wave front spread slowly through muscular conduction, mainly to the left and slightly forward. The constructed QRS complex showed a small negative delta wave in lead $\mathrm{V}_{1}$. Then, it entered into the Purkinje network in the right ventricular endocardium and spread across the interventricular septum from right to left nearly in parallel with the right septal surface. The activation sequence of the right ventricular free wall was not quite different from the normal one. The anterior wall of the left ventricle was excited by the normally conducted impulse, and the posterior region was activated last. The constructed QRS complex in lead $V_{1}$ showed a QS pattern with a negative delta wave, which was compatible with a type-C WPW complex proposed by Ueda et al. ${ }^{20}$ 

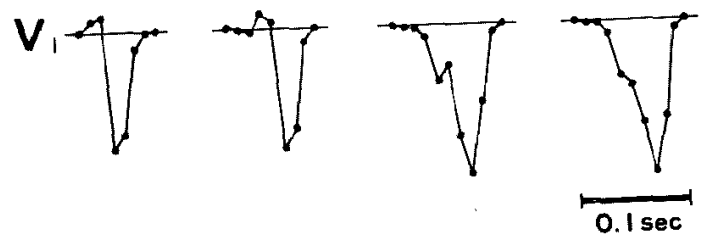

Fig. 6. QRS complexes in lead $V_{1}$ at various pre-excitation time with pre-excitation at the same location as in Fig. 5. The second and third QRS complexes from the left indicate fusion beats with the respective pre-excitation time of 20 and $40 \mathrm{msec}$. Irrespective of the degree of pre-excitation, they always show the type-G QRS complexes.

See legend of Fig. 2 for the left and right QRS complexes.

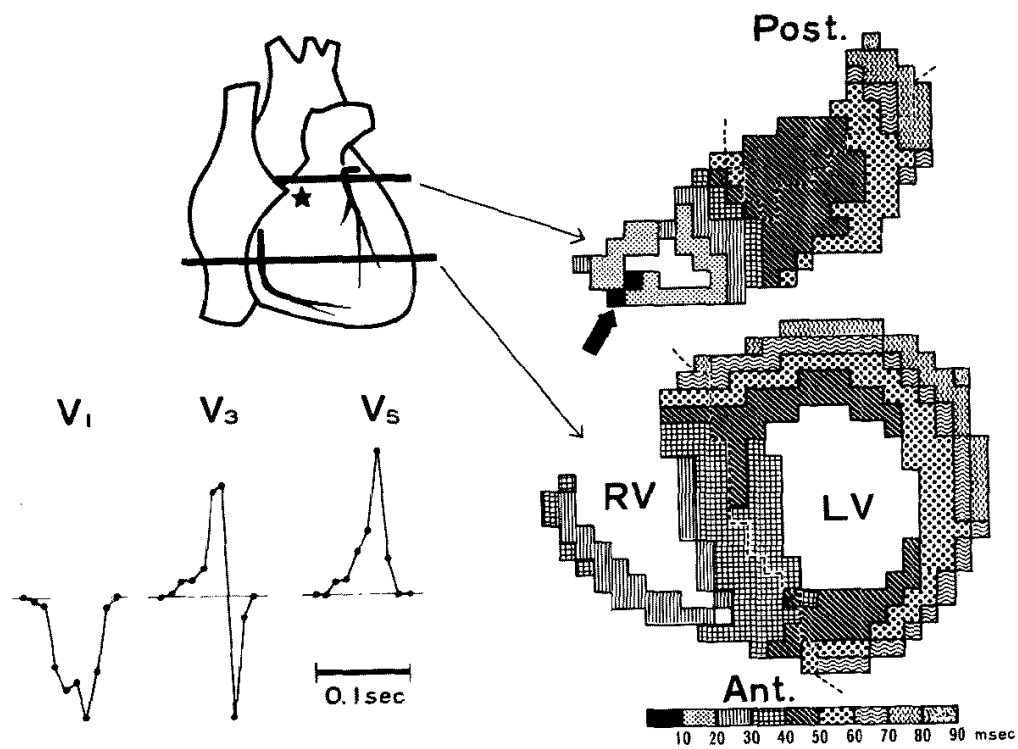

Fig. 7. Computer-simulated sequence of ventricular activation in preexcitation at the pulmonary conus. The site indicated by arrow in the right upper panel was excited $30 \mathrm{msec}$ earlier than the onset of normal excitation. The simulated QRS complexes are those of type $\mathrm{C}$. The lead $\mathrm{V}_{1}$ shows a QS pattern with a small negative delta wave.

See legend of Figs. 1 and 3 for the explanation of the broken lines and the ventricular activation wave front, respectively.

As pre-excitation time decreased, the QRS duration was shortened gradually, but the direction of the delta vector remained almost unchanged (Fig. 6). Irrespective of the degree of pre-excitation, the QRS complex consistently showed a QS pattern in lead $V_{1}$.

3. The pre-excitation impulse was given to the pulmonary conus 30 msec earlier than the normal excitation (Fig. 7). Initially (0 to $20 \mathrm{msec}$ ), the excitation wave front spread slowly through muscular conduction posteriorly 
slightly to the left and ensued in a small negative delta wave in lead $V_{1}$. Then, it invaded into the Purkinje network in the right ventricular endocardium $20 \mathrm{msec}$ after the onset of QRS and spread rapidly posteriorly to the right, with the interventricular septum activated from right to left. The apex and the posterior left ventricle were activated last (at 80 to $90 \mathrm{msec}$ ). The resultant QRS complex in lead $V_{1}$ showed a QS pattern, which was consistent with the type-C WPW syndrome. Irrespective of the degree of preexcitation, the ventricular fusion beat invariably showed a type-G WPW complex.

4. When the pre-excitation was set at the upper part of the anterior interventricular sulcus, the constructed QRS complex in lead $V_{1}$ also showed a QS or $\mathrm{W}$ configuration with a negative delta wave. Irrespective of the degree of pre-excitation, a type-C WPW complex was always obtained.

\section{Discussion}

\section{Pre-excitation at the postero-basal region of the left ventricle}

In 1944, Öhnell13) reported pathoanatomically of a patient with the WPW syndrome who had a muscle bundle connecting the left atrium with the left ventricle, located dorsally to the mitral ostium and peripherally to annulus fibrosus. Recently, Boineau ${ }^{(1)}$ and Wallace ${ }^{8)}$ and their coworkers reported epicardial mapping studies in patients with the type-A WPW syndrome which revealed early anomalous ventricular excitation at the posterior aspect of the left ventricle just beneath the A-V groove. On the basis of these facts, the pre-excitation stimulus was given at the subepicardial layer of the left ventricular postero-basal region to simulate type-A QRS complexes. QRS waves characteristic of type A were obtained when pre-excitation time was not short. The simulated ventricular activation sequence in the present study seems compatible with the findings in epicardial mapping studies on the human heart with the type-A WPW syndrome ${ }^{6)-8)}$ and also in animal experiments in which epicardial electrical stimulation of the posterior base of the left ventricle resulted in type-A QRS complexes. ${ }^{16)}$

When a type-A QRS alternates with a type-B one in a patient, it is generally supposed that he has more than one accessory bundle. ${ }^{20}$ In fact, Boineau et $\mathrm{a}^{6)}$ demonstrated the presence of biventricular pre-excitation in a squirrel monkey which had 2 anomalous atrioventricular muscular connections. The co-existence of 2 or more accessory bundles was also clinically suggested in several cases with the WPW syndrome. ${ }^{21,22)}$ Pointing out that the positive delta wave in the precordial leads remained unchanged in both type-A and -B QRS complexes in a case published by Ramachandran, ${ }^{22)}$ 
Bruyneel ${ }^{23)}$ mentioned that it would be hard to accecpt a change in the anatomic site of pre-excitation without altered initial vectors. In the present study, when pre-excitation time shortened, QRS complexes changed from type-A to type-B QRS complex (Fig. 2). This observation was compatible with the result of animal experiments reported by Iguchi. ${ }^{16)}$ The clinical counterpart of this observation is "concertina effect."13) Wyndham and colleagues ${ }^{24)}$ demonstrated that epicardial mapping identified early activation of the posterobasal portion of the left ventricle just to the left of the interventricular sulcus in a patient with type-B pre-excitation. The increase in atrioventricular nodal conduction time with atrial pacing preferentially favored conduction through the anomalous pathway with accentuation of preexcitation with the resulting QRS complexes being typical of type-A preexcitation in this patient.

\section{Pre-excitation at the antero-lateral base of the right ventricle}

Wood and colleagues ${ }^{25}$ first presented histologic proof of an accessory muscular connection at the right lateral border between the right atrium and ventricle in a patient with the WPW syndrome, as Kent ${ }^{26)}$ described in a variety of mammalian species. Earliest epicardial excitation was often observed at the right lateral border in patients with the type-B WPW syndrome. ${ }^{1)-4)}$ In the present study, a ventricular fusion beat with pre-excitation at the right lateral border neighboring the atrioventricular groove resulted in type-B QRS complexes (Fig. 3).

\section{Pre-excitation at the posterior paraseptal region of the right ventricle}

Ueda and coworkers ${ }^{27}$ ) suggested that the pre-excitation area was located at the base of the right ventricle in patients with the type-C WPW syndrome in which the delta vectors and QRS loops directed straight to the left. When the postcrior aspect of the right ventricle was prematurely activated in an experimental animal, a type- $\mathrm{G}$ ventricular fusion beat was observed. ${ }^{16)}$ Yamada et $\mathrm{al}^{12}$ have recently presumed that the site of pre-excitation was the posterior paraseptal region of the right ventricle in type III of the isopotential body-surface mapping. The results of the present simulation study were consistent with these reports. Independent of the degree of pre-excitation, the constructed QRS complexes in this instance remained to be of type-C configuration (Fig. 6).

Of interest is the fact that an unsuccessful case of attempted surgical interruption of the accessory pathway showed type-C QRS complexes (Figs. 1,2 , and 3 published by Lindsay et $\left.\mathrm{al}^{111}\right)$. In their case the earliest appearance of epicardial excitation was found at the lateral and posterior diaphrag- 
matic portion of the right ventricle. Lev and his associates ${ }^{28)}$ demonstrated A-V junctional bypass fibers and unusually copious Mahaim fibers from the $\mathrm{A}-\mathrm{V}$ bundle to the posterior portion of the muscular ventricular septum in a case of pre-excitation syndrome whose ECG had a short PR interval and delta waves and showed type-C QRS complexes (QS and $q R$ s in lead $V_{1}$ ).

Pre-excitation at the pulmonary conus and the upper part of the anterior interventricular sulcus

When pre-excitation started at the subepicardial layer of the pulmonary conus (Fig. 7) or of the upper part of the anterior interventricular sulcus, type-C QRS complexes were obtained irrespective of the degree of pre-excitation. However, no clinical reports have been made which demonstrated pathoanatomically and suggested electrophysiologically the presence of anomalous atrioventricular communications in these areas. It would be unlikely on the embryological basis that the anomalous A-V pathway enters the pulmonary conus.

\section{ACKNOWLEDGEMENTS}

The author expresses sincere gratitude to Prof. Itsuro Sobue and Prof. Kazuo Yamada for their helpful advice, and to Prof. Mitsuharu Okajima and Dr. Iwao Sotobata for their kind guidance and suggestion throughout the study. The author is also indebted to Dr. Shoji Yasui for his valuable discussion.

\section{REFERENCES}

1. Durrer D, Roos JP: Epicardial excitation of ventricles in patient with Wolff-ParkinsonWhite syndrome (type B). Circulation 35: 15, 1967

2. Burchell HB, Frye RL, Anderson MW, McGoon DC: Atrioventricular and ventriculoatrial excitation in Wolff-Parkinson-White syndrome (type B). Circulation 36: 663, 1967

3. Cobb FR, Blumenschein SD, Sealy WC, Boineau JP, Wagner GS, Wallace AG: Successful surgical interruption of the bundle of Kent in a patient with Wolff-Parkinson-White syndrome. Circulation 38: 1018, 1968

4. Iwa T, Kazui T, Sugii S, Wada J: Wolff-Parkinson-White syndrome. Surgical trcatment. Jap J Thorac Surg 23: 513, 1970

5. Boineau JP, Moore EN: Evidence for propagation of activation across an accessory atrioventricular connection in types A and B preexcitation. Circulation 41:375, 1970

6. Boineau JP, Moore EN, SpearJF, Sealy WG: Basis of static and dynamic electrocardiographic variations in Wolff-Parkinson-White syndrome. Am J Cardiol 32: 32, 1973

7. Neutze JM, Kerr AR, Whitlock RML: Epicardial mapping in a variant of type A WolffParkinson-White syndrome. Circulation 48:662, 1973

8. Wallace AG, Sealy WC, Gallagher JJ, Svenson RH, Strauss HC, Kasell J: Surgical correction of anomalous left ventricular pre-excitation. Wolff-Parkinson-White (type A). Circulation 49: 206, 1974

9. Lister JW, Worthington FX, Gentsch TO, Swenson JA, Nathan DA, Gosselin AJ: Pre- 
excitation and tachycardias in Wolff-Parkinson-White syndrome, type B. Circulation 45: 1081,1972

10. Cole JS, Wills RE, Winterscheid LG, Reichenbach DD, Blackmon JR: The Wolff-ParkinsonWhite syndrome. Problems in evaluation and surgical therapy. Circulation 42: 111, 1970

11. Lindsay AE, Nelson RM, Abildskof JA, Wyatt R: Attempted surgical division of the preexcitation pathway in the Wolf-Parkinson-White syndrome. Am J Cardiol 28: 581, 1971

12. Yamada K, Toyama J, Wada M, Sugenoya J, Toyoshima H, Mizuno Y, Sotobata I, Kobayashi $T$, Okajima $M$ : Body surface isopotential mapping in Wolff-Parkinson-White syndrome. Non-invasive method to determine the localization of the accessory atrioventricular pathway. Am Heart J 90: 721, 1975

13. Öhnell RF: Pre-excitation, a cardiac abnormality. Acta Med Scand 152 (suppl): 1, 1944

14. Butterworth JS, Poindexter CA: Fusion beats and their relation to the syndrome of short P-R interval associated with a prolonged QRS complex. Am Heart J 28: 149, 1944

15. Moore EN, Spear JF, Boineau JP: Electrophysiological studies on preexcitation in the dog using an electronically simulated atrioventricular bypass pathway. Circulat Res 31: 174, 1972

16. Iguchi K: Experimental study on fusion beat with special reference to WPW syndrome. Tokyo J Med Sc 76: 344, 1968 (in Japanese)

17. Okajima M, Fujino T, Kobayashi T, Yamada K: Computer simulation of the propagation process in excitation of the ventricles. Circulat Res 23: 203, 1968

18. Okajima M, Fujino T, Muraki H, Kobayashi T, Yamada K: Computational reconstruction of electrocardiographic QRS pattern. By means of simulated ventricular propagation processes of excitation and transfer impedance values. Heart 1: 138, 1969 (in Japanese)

19. Rosenbaum FF, Hecht $\mathrm{HH}$, Wilson FN, Johnston FD: The potential variations of the thorax and the esophagus in anomalous atrioventricular excitation (Wolff-Parkinson-White syndrome). Am Heart J 29; 281, 1945

20. Ueda H, Nameki C, Saruta H, Kawamura H, Yoshida A, Tsuzuku A: Further studies on the W.P.W. syndrome (preexcitation syndrome) with special reference to the intracardiac and esophageal lead. Jap Circulat J 21: 361, 1957

21. Hecht HH, Kennamer R, Prinzmetal M, Rosenbaum FF, Sodi Pallares D, Wolff L, Brooks C, Pick A, Rijland P, Robb JS: Anomalous atrioventricular excitation. Panel discussion. Ann New York Acad Sc 65: 826, 1957

22. Ramachandran S: Wolff-Parkinson-White syndrome. Conversion of type A to type B electrocardiographic changes. Circulation 45: 529, 1972

23. Bruyneel KJJ; Wolff-Parkinson-White syndrome. Circulation (correspondence) 47: 433, 1973

24. Wyndham CRC, Amat-y-Leon FL, Denes P, Dhingra RC, Burman SO, Pouget JM, Rosen KM: Posterior left ventricular preexcitation. Report of a case. Arch Intern Med 134: 243,1974

25. Wood FC, Wolferth GC, Geckeler GD: Histologic demonstration of accessory muscular connections between auricle and ventricle in a case of short P-R interval and prolonged QRS complex. Am Heart J 25: 454, 1943

26. Kent AFS: Researches on the structure and function of the mammalian heart. J Physiol 14: 233,1893

27. Ueda H, Harumi K, Shimomura K, Yamamoto H, Sugimoto T: A vectorcardiographic study of WPW syndrome. Jap Heart J 7: 255, 1966

28. Lev $M$, Leffler WB, Langendorf $R$, Pick A: Anatomic findings in a case of ventricular preexcitation (WPW) terminating in complete atrioventricular block. Circulation 34: 718, 1966 